\title{
AN ELASTO-VISCOUS MODEL OF THE EARTH
}

\author{
S. Losito*, B. Pernice, D. Picca, G. Verrone \\ *IESI-CNR \\ Dipartimento di Fisica \\ Via Amendola 173 \\ 70126 Bari, Italy
}

\begin{abstract}
A two-symmetric-rigid-rotators model of the Earth has been studied, under the hypothesis of elasto-viscous coupling. The free Eulerian equation of motion has been solved in the linear approximation related to small wobbling amplitudes. Under these hypotheses, polar motion is stable, and the angular velocity of the Earth is the sum of three vectors rotating with different frequencies and damped amplitudes. One of these terms turns out to be retrograde with a quasidiurnal frequency and could be identified with a similar term appearing in liquid core models of the Earth. The other two terms are identified with the Chandler wobble and the annual term according to observational data. The elastic coupling produces, in the time variation of L.O.D., a periodic term whose frequency is about one year. It could be hypothesized that the "decade fluctuation" could be partially attributed to the free oscillation of the Earth.
\end{abstract}

\section{THE MODEL}

The problem of polar motion has not been well modeled until now. We have studied an elasto-viscous model of the Earth under some simplifying hypotheses. Detailed calculations will appear elsewhere. Here we outline the main results. Two rigid symmetric rotators are considered, the outer for the crust-mantle and the inner for the core. We adopt two reference frames, with the same origin, $\left(\bar{x}_{1}, \bar{x}_{2}, \bar{x}_{3}\right)$ for the mantle and $(\bar{a}, \bar{b}, \bar{c})$ for the core. It is assumed also that the elastic coupling between the two rotators can be expressed as $T=\sigma \cdot \alpha$ where $\sigma$ is a positive constant and represents the angular deviation of the core from its equilibrium position with respect to the mantle.

The viscous coupling is assumed to be proportional to the difference between the angular velocity of the core $\dot{\bar{\omega}}$ and the mantle $\bar{\Omega}$, via a second order tensor of inertia $\overleftrightarrow{u}$. The Eulerian equations of motion for the mantle and the core give rise to four differential equations for $\dot{\bar{\Omega}}, \dot{\bar{\omega}}, \dot{\bar{c}}, \dot{\bar{a}}$. The total torque considered is the sum of the elastic torque, the viscous torque, and a residual torque, which include all the external interactions of the systems. 


\section{FREE MOTION SOLUTIONS}

To solve this set of differential equations, we adopt for $\bar{\Omega}, \bar{\omega}, \bar{c}, \bar{a}$ the hypothesis of small wobbling amplitudes, well supported by observations.

The Free Motion Solution, that is, the equation with no external interactions, can be split into two new equations; one differential equation is referred to as the free equation of L.O.D. owing to the fact that its most representative term is the parameter $m_{3}$ related to $\Delta \Omega / \Omega_{0}$ where $\Omega_{0}=1 \mathrm{rev} /$ day is the mean angular velocity of the Earth.

The second equation is referred to as the Free Polar Motion Equation (FPME), owing to the fact that it contains the parameters $m_{1}$ and $m_{2}$ of the angular velocity of the Earth in the equatorial plane $\left(\bar{x}_{1}, \bar{x}_{2}\right)$ that is the wobbling components.

From the FPME one finds that the $m_{1}(t)$ and $m_{2}(t)$, parameters of the wobbling components of $\bar{\Omega}$, are the sum of three rotating vectors, each in the equatorial plane $\left(\bar{x}_{1}, \bar{x}_{2}\right)$, each with its frequency and each with damped amplitudes.

The first two complex eigenvalues of the solution are identified respectively with the annual term and the Chandler term of the Earth rotation; the third eigenvalue is related to a term of period $T_{d}=-.999991$ days and damped time $\tau_{d}=.4693713$ years. That is to say the third term turns out to be a retrograde diurnal term.

It is also possible to obtain an estimate of the nondimensional parameters related to the elastic and viscous coupling. The main term $m_{3}(t)$ of the F.E. of L.O.D. contains oscillating terms with frequency depending on the viscous parameter. Setting this parameter to zero, one obtains a frequency $=19.1910$ $\mathrm{rad} /$ day with a period of 327 days.

\section{SUMMARY}

The annual term is usually related to the interaction of the Earth with the atmospheric angular momentum. In this work it is assumed as a characteristic of the Free Motion. The quasi-diurnal retrograde term has been found for liquid core models. In this work it is derived from a solid core model.

The annual term variation in the L.O.D. is an indication that the "decade fluctuations" in the L.O.D. can be partly attributed to the viscous coupling between the core and the mantle.

\section{REFERENCES}

Flodmark, S., and Davstad, K., "Core-Mantle Interaction and Chandler Wobbles," Earth's Rotation: Solved and Unsolved Problems, NATO Advances Research Workshop, Chateau de Bonas, Gers, France, 1985.

Kubo, Y., "An Explanation of the Polar Motion by a Rigid Core-Mantle Model," in Rotation of the Earth, Melchior, P., and Yumi, S., eds., copyright IAU, 1982, p. 182.

Lambeck, K., The Earth Variable Rotation: Geophysical Causes and Consequences, Cambridge University Press, Capt. I, 1980.

Rochester, M. G., "The Earth's Rotation," EOS (Trans. Am. Geophysical Union), 54, 769-780, 1973. 